\title{
ANÁLISIS DE LOS INDICADORES ECONÓMICOS DE LA OCDE SOBRE LOS SISTEMAS DE PENSIONES EN MÉXICO
}

Ana Bertha Vidal Fócil*, Juan Menéndez Blanco**, Luis Manuel Hernández Govea***

* Doctora en Economía. Directora General de Modernización Administrativa en la Coordinación de Modernización Administrativa e Innovación Gubernamental del Gobierno del Estado de Tabasco. Universidad Juárez Autónoma de Tabasco.

** Profesor Investigador de la Facultad de Ciencias Económicas y Empresariales. Universidad Complutense de Madrid.

*** Profesor Investigador de la División Académica de Ciencias Económico Administrativas de la Universidad Juárez Autónoma de Tabasco. Universidad Juárez Autónoma de Tabasco.

Dirección para recibir correspondencia: berthafocil@hotmail.com 


\section{RESUMEN}

Objetivo: Analizar los indicadores que la Organización para la Cooperación y el Desarrollo Económico (OCDE), ha presentado en cuanto al Sistema de Pensiones de México, para comprender el impacto que ha tenido la reforma en la solución de los problemas de pobreza y desigualdad en la que viven los adultos mayores; y así establecer consideraciones para elevar la eficiencia.

Material y método: La investigación es de tipo descriptivo, ya que se realizará un análisis económico del Sistema de Pensiones de México, realizando un estudio de los indicadores de la OCDE que presenta en su informe "Global Pension Statistics". Para el análisis se realizaron tablas y figuras que describen datos del 2000 al 2013.

Resultados: El gasto público en pensiones representa la principal partida del gasto en protección social, del 2005 al 2008 se tuvo un incremento en los fondos de pensiones desde el punto de vista del porcentaje, que representan del Producto Interno Bruto (PIB). Los fondos de pensiones se han invertido en bonos públicos y privados, con una composición similar desde 2007, año en que se dio una apertura en cuanto a los instrumentos en que podían ser invertidos estos fondos. Los fondos de pensiones, tanto públicos como privados han tenido en México un crecimiento relevante.

Conclusiones: El Estado debe disminuir la economía laboral sumergida, permitiendo que el paso a la legalidad sea sencillo y estos trabajadores y sus familias tengan estímulos para adherirse al sistema de Seguridad Social. Si no se realizan las acciones necesarias, México puede entrar en una crisis financiera por el funcionamiento actual del sistema de pensiones.

Palabras Clave: OCDE. Pensiones. México, AFORE. 


\section{ABSTRACT}

Objective: Analyze indicators that the Organization for Economic Cooperation and Development has presented concerning the Pension System in Mexico to understand the impact that reform has had in solving the problems of poverty and inequality in which older adults live; and thus establish considerations to increase efficiency.

Material and method: The research is descriptive and an economic analysis of the Mexican pension system will be made, making a study of OECD indicators presented in its report "Global Pension Statistics". For the analysis charts and graphs that describe data from 2000 to 2013 were performed.

Results: Public pension expenditure represents the main item of social protection expenditure from 2005 to 2008 had been an increase in the pension funds from the point of view of the percentage they represent of the Gross Domestic Product. Pension funds are invested in government and corporate bonds with a similar composition since 2007, when an opening occurred in terms of instruments that could be invested in these funds. Pension funds, both public and private have had significant growth in Mexico.

Conclusions: The State should reduce the underground labor economy, allowing the legality step simple and these workers and their families have incentives to join the social security system. If the necessary actions Mexico may enter into a financial crisis for the current functioning of the pension system are not made.

Key Words: OCDE. Pensions. México. AFORE. 
En México, la reforma moderna del Sistema de Pensiones se inició en el año 1992 con la instauración del Sistema de Ahorro para el Retiro (SAR), programa que complementó a lo ya generado, con aportación del $2 \%$ del Salario Base de Cotización (SBC) para el retiro y el $5 \%$ para vivienda; recursos que se contabilizaban de forma individual y se acumulaban en el Banco Central. Sin embargo, la reforma del Sistema de Pensiones de los trabajadores del sector formal privado administrada por el IMSS, fue aprobada en diciembre de 1995, entrando en vigor el primero de julio de 1997. En el año de 1995, México enfrentaba una importante crisis económica, ya que un año antes el peso sufrió un ataque especulativo, con consecuencias significativas en la fuga de capitales, a lo que le siguió la devaluación del peso. En este proceso que vivió México, el ahorro externo altamente volátil era uno de factores negativos a resolver. En 1995, la Presidencia de la República de Ernesto Zedillo Ponce de León propuso como objetivo central en su plan nacional la promoción del ahorro interno, y, en particular, del privado, como una fuente de financiamiento con estabilidad y permanencia de la inversión. En este contexto se discutió la reforma como un mecanismo para coadyuvar en la búsqueda de estos objetivos.

La reforma del IMSS supuso la sustitución del esquema de reparto y beneficios definidos por uno de capitalización total, basado en cuentas individuales. Pese a que el cambio se dio de manera obligatoria, los trabajadores ya pensionistas no modificaron su situación, y los trabajadores activos al momento de la reforma tienen la alternativa de elegir en el momento de su retiro, los beneficios del nuevo régimen o continuar con el anterior. El diseño del nuevo sistema de pensiones está fundamentado en tres características elementales; financiación mediante un esquema de capitalización, administración de cuentas individuales por empresas especializadas de giro exclusivo y libre elección del trabajador; ésta última se puede dar de 3 formas distintas: elección de la administradora de fondos de pensiones para el manejo de su cuenta de ahorro para el retiro, manejo y control del fondo en su cuenta de ahorro para el retiro a través de los estados de cuenta y de la elección del fondo de inversión con la mezcla de riesgo y rendimiento que más se acerque a las preferencias del trabajador, y elección de la modalidad de pensiones la cual puede ser en forma de una renta vitalicia adquirida con alguna aseguradora o un retiro programado con una administradora de fondo de pensiones.

\section{Regulación del sistema de pensiones después de la reforma}

En lo que respecta a la regulación de este sistema, la Comisión Nacional del Sistema de Ahorro para el Retiro (CONSAR) -organismo responsable de la regulación y supervisión 
de las operaciones vinculadas con el sistema reformado de pensiones- es la encargada de emitir las normas sobre las cuales deben de someterse los ofertantes y demandantes. El sistema reformado es de pilares múltiples: cuenta con un pilar básico de beneficios definidos a través de una pensión mínima garantizada, igual al salario mínimo vigente el 1 de julio de 1997,que se actualiza cada año en febrero de acuerdo con la variación del Índice Nacional de Precios al Consumidor (INPC). El trabajador o usuario tiene derecho a esta pensión mínima siempre y cuando haya cotizado 1,250 semanas y si el saldo acumulado en su cuenta individual es inferior al necesario para gozar de una renta vitalicia o llevar a cabo un retiro programado mayor o igual a la pensión mínima garantizada.

La cuenta individual de cada trabajador se compone de tres subcuentas:

1. Retiro, cesantía en edad avanzada y vejez (RCV).

2. Vivienda.

3. Aportaciones voluntarias.

La aportación a la subcuenta de retiro, cesantía en edad avanzada y vejez (RCV), es igual al $6.5 \%$ del SBC del trabajador más una aportación.

Al retiro, los trabajadores o usuarios tienen el derecho de disponer de los recursos acumulados en su cuenta, a través de retiros programados o adquiriendo una renta vitalicia. Los retiros programados son administrados por la misma administradora, mientras que las rentas vitalicias tienen que ser contratadas con una aseguradora autorizada. Por lo que respecta a los retiros programados, el trabajador asume el riesgo de sobrevivir el periodo programado para el pago de su pensión, así como el riesgo que implican los cambios en la tasa de interés; para las rentas vitalicias, la aseguradora cobra una prima por garantizar al trabajador una suma predeterminada por concepto de pensiones hasta la muerte del asegurado o sus beneficiarios (Villagomez \& Hernández, 2010; Sales, Solís, \& Villagómez, 1997; Solís \& Santín, 1999).

En cuanto al ISSSTE -su nueva ley fue publicada el 31 de marzo de 2007 en el Diario Oficial de la Federación- considera reformas similares a las realizadas por el IMSS en materia de pensiones, ya que se sustituyó el sistema de reparto o beneficios que, definidos por el de capitalización individual (contribuciones definidas), será la base para 
todas las pensiones; se establece la instalación del Fondo Nacional de Pensiones de los Trabajadores al Servicio del Estado (PENSIONISSSTE) y criterios para la portabilidad de derechos con el instituto. En relación con el nuevo Sistema de Pensiones de retiro, los trabajadores en activo pueden elegir entre mantenerse en el sistema anteriormente utilizado o entrar al nuevo; en cuanto que los de nuevo ingreso se incorporaran al nuevo sistema.

En el nuevo sistema, los trabajadores deberán abrir una cuenta individual y se les otorgará un bono de pensión, el cual es un título de deuda emitido por el gobierno federal a cuenta de lo aportado en el sistema de pensiones anterior a la reforma de 2007, este bono se hará efectivo en el momento de adherirse al sistema. Este fondo será administrado por PENSIONISSSTE durante 36 meses; después de este plazo los trabajadores elegirán si continuar o cambiar a una AFORE. Al igual que en el IMSS, esta cuenta se conformará por las subcuentas de retiro, cesantía y vejez (RCV); fondo de vivienda, ahorro solidario, aportaciones complementarias de retiro, aportaciones voluntarias y ahorro a largo plazo.

En este esquema se crea el ahorro solidario, en el que el trabajador optará por el descuento de hasta el $2 \%$ de su sueldo básico para aportarlo en su cuenta individual; en apremio de lo anterior, las dependencias y entidades depositarán 3.25 pesos por cada peso ahorrado por el usuario hasta el 6.5\% del sueldo básico. Las pensiones a recibir por el trabajador bajo el nuevo sistema son cesantía en edad avanzada y vejez; para la primera se requerirá tener 60 años de edad y estar privado de trabajo; en tanto que para la segunda se requerirá tener 65 años de edad, 5 años más que en el régimen anterior, además de haber cotizado 25 años.

En lo que respecta a las modalidades para adherirse al Sistema de Pensiones, éstas son las mismas que operan en el IMSS, en la que existe la modalidad de renta vitalicia, llamada seguro de pensión con seguro de sobrevivencia y el retiro programado. Del mismo modo, está en vigor la pensión garantizada para los trabajadores que, cumpliendo con los requisitos, sus recursos sean insuficientes para adquirir el seguro de pensión; a razón del doble salario mínimo, la pensión anticipada y retiros de la cuenta individual antes de convertirse en pensionista. 
Por último, el PENSIONISSSTE no podrá cobrar una comisión que exceda el promedio de las que se cobran en las AFORE's. Las cuotas por el seguro de retiro, cesantía en edad avanzada y vejez (RCV) correspondientes a los trabajadores se ajustaran año con año a partir de la entrada en vigor de la nueva legislación, del 3.5\% hasta llegar al $6.125 \%$ en un periodo de 5 años entre 2007 y 2012 (Morales, 2007).

\section{RESULTADOS}

La OCDE, mencionó en el 2013 que México tenía la tasa de pobreza más alta entre los países miembros de esta organización, después de Australia y Corea. Al finalizar la década del 2000 , el $27.6 \%$ de las personas de 65 años en adelante se encontraban en riesgo de entrar en situación de pobreza. Este organismo internacional plantea que los adultos mayores con edades más altas tienen mayor vulnerabilidad (OCDE, 2013).

A continuación, se analizarán diversos indicadores realizados por la OCDE, que permiten estudiar el panorama de las pensiones en México. La tasa de reemplazo es un indicador de fácil comprensión y cálculo que muestra si la cuantía de las pensiones a recibir es adecuada con las expectativas de consumo de los adultos mayores, la cual se obtiene al dividir la pensión entre el salario obteniendo una proporción que se expresa en porcentaje. La OCDE, recomienda utilizar el concepto de Tasa de Reemplazo Bruta como la pensión bruta dividida entre el salario antes del retiro (este indicador considera los impuestos para estos ingresos).

VIDAL-FÓCIL A.B., MENÉNDEZ-BLANCO J., HERNÁNDEZ-GOVEA L.M. 
Tabla 1

Tasa de reemplazo de pensiones en México para hombres y mujeres y promedio de la OCDE, en el periodo 2005-2013

\begin{tabular}{|c|c|c|c|}
\hline Año & $\begin{array}{l}\text { Tasa bruta de reemplazo } \\
\text { en México en Hombres } \\
\text { (Ingreso promedio) }\end{array}$ & $\begin{array}{l}\text { Tasa bruta de reemplazo } \\
\text { en México en Mujeres } \\
\text { (Ingreso promedio) }\end{array}$ & $\begin{array}{l}\text { Tasa bruta de } \\
\text { reemplazo promedio } \\
\text { de los países de la } \\
\text { OCDE (Ingreso } \\
\text { promedio) }\end{array}$ \\
\hline 2005 & $36.0 \%$ & $21.7 \%$ & $57.2 \%$ \\
\hline 2007 & $35.8 \%$ & $29.7 \%$ & $58.7 \%$ \\
\hline 2009 & $36.9 \%$ & $32.5 \%$ & $60.8 \%$ \\
\hline 2011 & $46.3 \%$ & $46.3 \%$ & $60.6 \%$ \\
\hline 2013 & $44.7 \%$ & $44.7 \%$ & $57.9 \%$ \\
\hline
\end{tabular}

Fuente: Elaboración propia con base en Pensions at a Glance: Retirement-Income Systems in OECD Countries para los años 2005, 2007, 2009, 2011 y 2013.

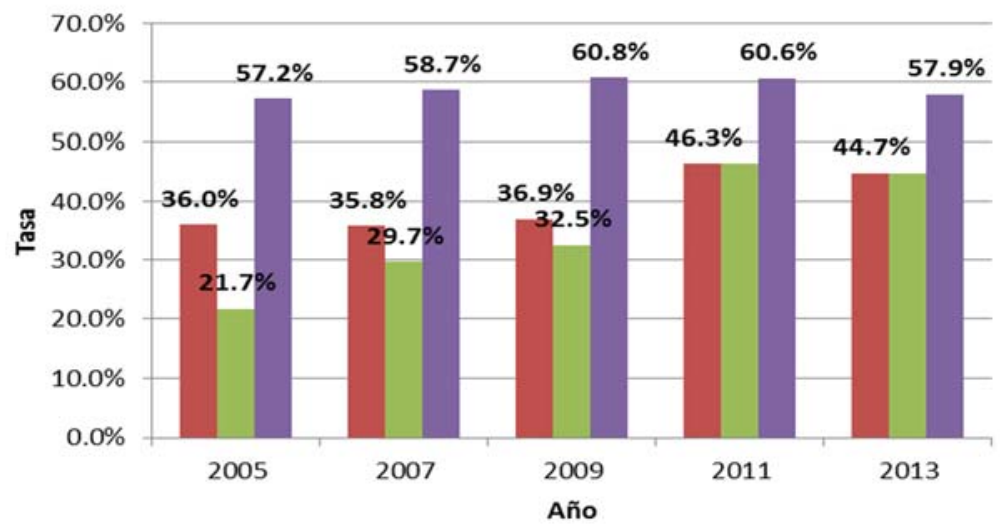

- Tasa bruta de reemplazo en México en Hombres (Ingreso promedio)

- Tasa bruta de reemplazo en México en Mujeres (Ingreso promedio)

- Tasa bruta de reemplazo promedio de los paises de la OCDE (Ingreso promedio)

Figura 1. Tasa de reemplazo de pensiones en México para hombres y mujeres y promedio de la OCDE, en el periodo 2005-2013.

Fuente: Elaboración propia con base en Pensions at a Glance: Retirement-Income Systems in OECD Countries para los años 2005, 2007, 2009,2011 y 2013. 
La óptima factibilidad de la tasa de reemplazo ideal podría situarse en el cien por ciento; sin embargo, hay que considerar que el consumo de este sector de la población cambia en el momento de la jubilación, ya que las necesidades se modifican. Los gastos en salud se incrementan y gastos como el transporte, vestimenta y educación tienden a bajar drásticamente. Por ello, diversos organismos e instituciones internacionales recomiendan que la tasa fluctué entre el 50 y el 70\%. La OCDE y la Organización Internacional del Trabajo (OIT), reconocen adecuado una tasa del 50\% (CONSAR, 2013).

Al examinar la figura de la tasa de reemplazo en México, lo primero que se observa es que está por debajo tanto del promedio de los países de la OCDE, como de la recomendada por éste. Aunque hasta antes del 2011 se incrementó, para el 2013 disminuyó en 1.6\%; además, la brecha de equidad con respecto al género ha disminuido, ya que desde el 2011 se ha mantenido el indicador en el mismo nivel tanto para hombres como para mujeres.

A lo anterior, hay que agregar que el Índice Nacional de Precios al Consumidor, genera que el poder adquisitivo de la población pensionista disminuya, ya que el monto de la pensión no aumenta como lo hace este indicador; por lo tanto, el poder adquisitivo real, como el verdadero nivel de consumo que puede tener una persona a un nivel de precios determinado disminuye (Centro de Estudios de las Finanzas Públicas, 2009). Para el periodo 2005-2013, el comportamiento del INPC es el siguiente:

VIDAL-FÓCIL A.B., MENÉNDEZ-BLANCO J., HERNÁNDEZ-GOVEA L.M. 
Tabla 2

Índice nacional de precios al consumidor en México, en el periodo 2005-2013

\section{INPC Anual en México}

\begin{tabular}{ll} 
Año & (\%) \\
\hline 2005 & 3.3 \\
2006 & 4.1 \\
2007 & 3.8 \\
2008 & 6.5 \\
2009 & 3.6 \\
2010 & 4.4 \\
2011 & 3.8 \\
2012 & 3.6 \\
$\mathbf{2 0 1 3}$ & 4.0
\end{tabular}

Fuente: Elaboración propia con base en OECD Main Economic Indicators and IMF International Financial Statistics; Banco de México.

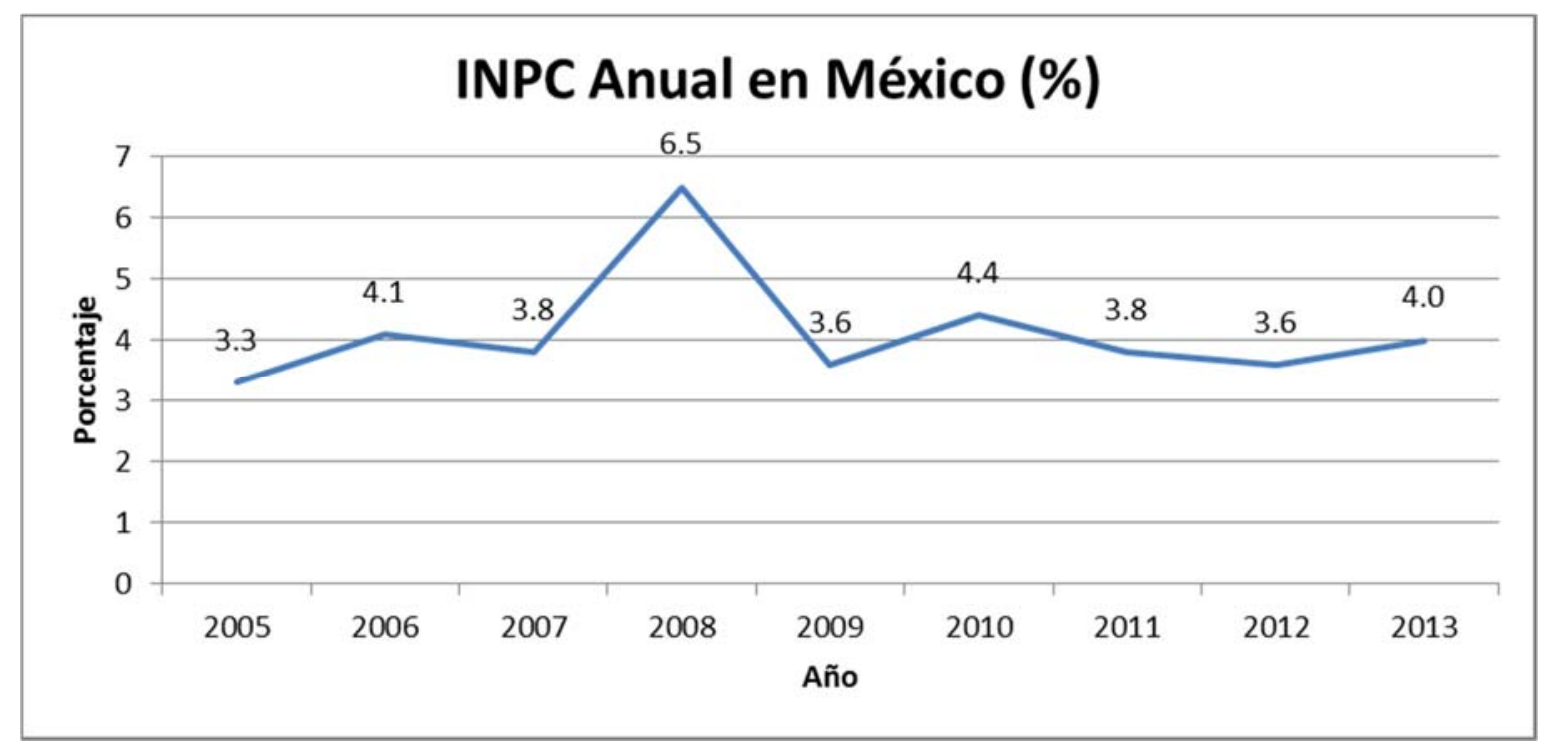

Figura 2. Índice nacional de precios al consumidor en México, en el periodo 2005-2013.

Fuente: Elaboración propia con base en OECD Main Economic Indicators and IMF International Financial Statistics; Banco de México. 
Aunque a partir del 2008, este indicador se ha mantenido menor al 5\%, es importante precisar el comportamiento de éste, ya que los programas no contributivos deben de plantearse con base en el comportamiento de los precios de los bienes y servicios demandados por los ancianos. Otro indicador de importancia para el análisis es el gasto en pensiones como porcentaje del PIB (Producto Interno Bruto).

Tabla 3

Gasto público en pensiones como porcentaje del PIB en México, en el periodo 2000-2013

\begin{tabular}{lll}
\hline & \multicolumn{2}{c}{ Gasto público en pensiones en } \\
& México (\% del PIB) & \\
\hline 2000 & $0.6 \%$ & $6.4 \%$ \\
2007 & $1.1 \%$ & $6.4 \%$ \\
2010 & $1.5 \%$ & $7.3 \%$ \\
2011 & $1.6 \%$ & $7.4 \%$ \\
2013 & $1.7 \%$ & $7.8 \%$
\end{tabular}

Fuente: Elaboración propia con base en sistema estadístico de la OCDE: OCDE. StatExtracts, 2014.

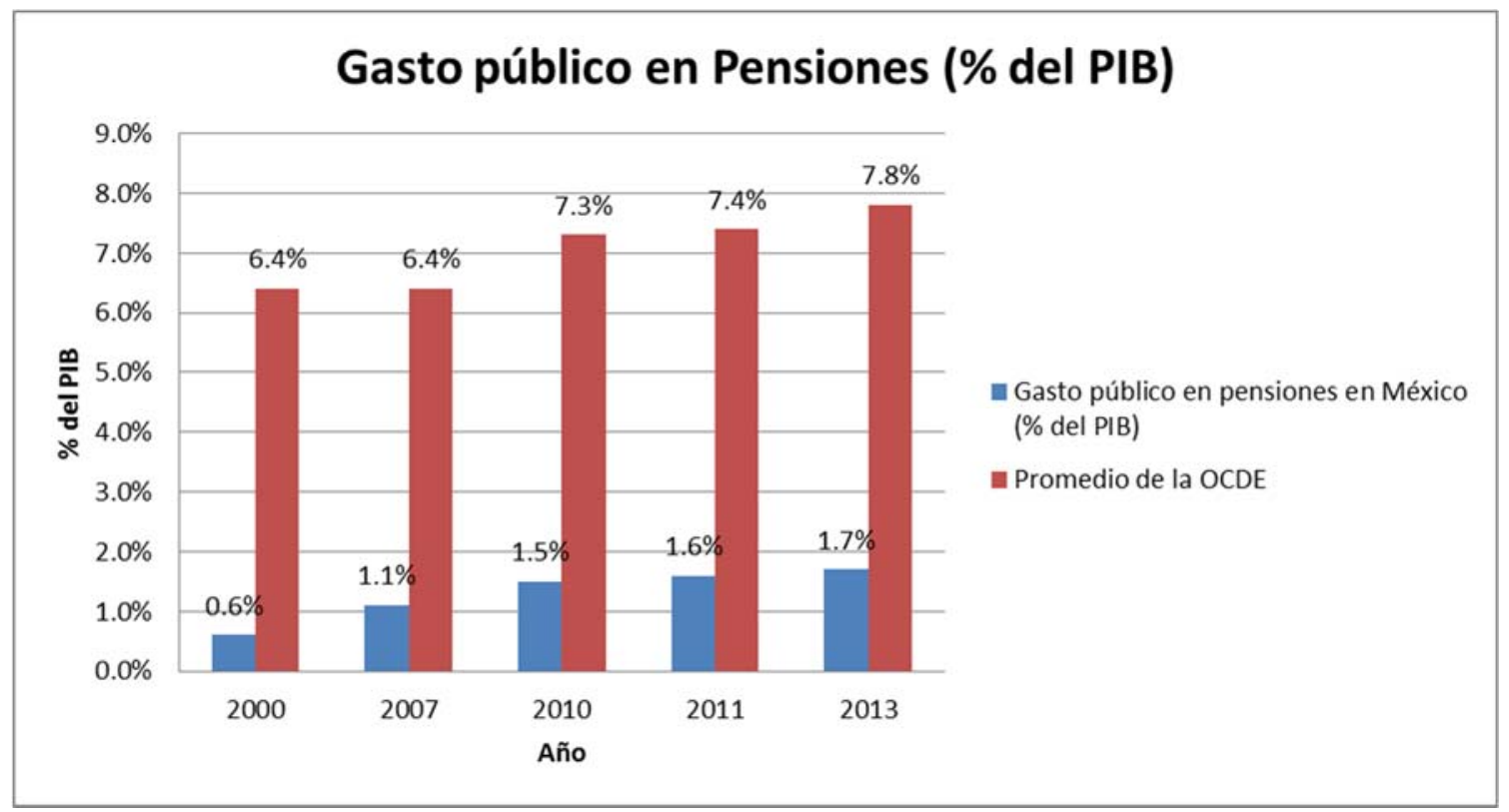

Figura 3. Gasto público en pensiones como porcentaje del PIB en México, en el periodo 2000-2013.

Fuente: Elaboración propia con base en sistema estadístico de la OCDE: OCDE. StatExtracts, 2014. 
El gasto público en pensiones, representa la principal partida del gasto en protección social. Las pensiones de jubilación son la principal partida dentro del gasto en pensiones. Un aumento del crecimiento del gasto público en pensiones como porcentaje del PIB puede deberse a una disminución del crecimiento de este último, o a un aumento en las obligaciones del gobierno en esta temática. El gasto en pensiones es importante que se ajuste al crecimiento del PIB, de modo que se busque la sostenibilidad de las finanzas públicas; en México este indicador ha tenido un incremento menos acelerado desde 2007 , año en el cual se realizó la reforma al sistema de pensiones del ISSSTE, y menor a los que presentan, en promedio, los países de la OCDE.

Como contraparte del gasto público en pensiones, se ubica las contribuciones al fondo de las pensiones. Un fondo de pensiones cuanto mayor sea, mayores serán las utilidades obtenidas de su inversión. En México, como se muestra en la tabla y grafica siguiente, del 2005 al 2008 se tuvo un incremento en los fondos de pensiones desde el punto de vista del porcentaje que representan del Producto Interno Bruto; sin embargo, desde ese año hasta el último dato analizado (2012), se observa que ha disminuido paulatinamente. Con este dato, y el anterior que describe el gasto público en pensiones, se observa que estamos en una situación deficitaria, donde existe un gasto público en pensiones mayor a los recursos obtenidos. 


\section{Tabla 4}

Contribuciones al fondo de pensiones en México (\% del PIB), durante el periodo 20012012.

\begin{tabular}{ll}
\hline Año & $\begin{array}{l}\text { Contribuciones al fondo de } \\
\text { pensiones en México (\% del } \\
\text { PIB), Periodo 2001-2012 }\end{array}$ \\
\hline 2001 & $0.99 \%$ \\
2002 & $0.83 \%$ \\
2003 & $0.78 \%$ \\
2004 & $0.75 \%$ \\
2005 & $0.75 \%$ \\
2006 & $0.95 \%$ \\
2007 & $0.81 \%$ \\
2008 & $1.19 \%$ \\
2009 & $0.95 \%$ \\
2010 & $0.98 \%$ \\
2011 & $0.95 \%$ \\
2012 & $0.87 \%$ \\
\hline
\end{tabular}

Fuente: Elaboración propia con base en OECD Global Pension Statistics, 2013. La interrupción de la serie en 2006 se debe a la inclusión de los planes de pensiones de empleo registrados por CONSAR desde 2005, no está incluido en los años anteriores. Las contribuciones totales incluyen las contribuciones obligatorias para la jubilación de los empleados, empleadores y el gobierno, y las contribuciones voluntarias y las transferencias del sistema de pensiones anterior (válido hasta el 1997).

VIDAL-FÓCIL A.B., MENÉNDEZ-BLANCO J., HERNÁNDEZ-GOVEA L.M. 


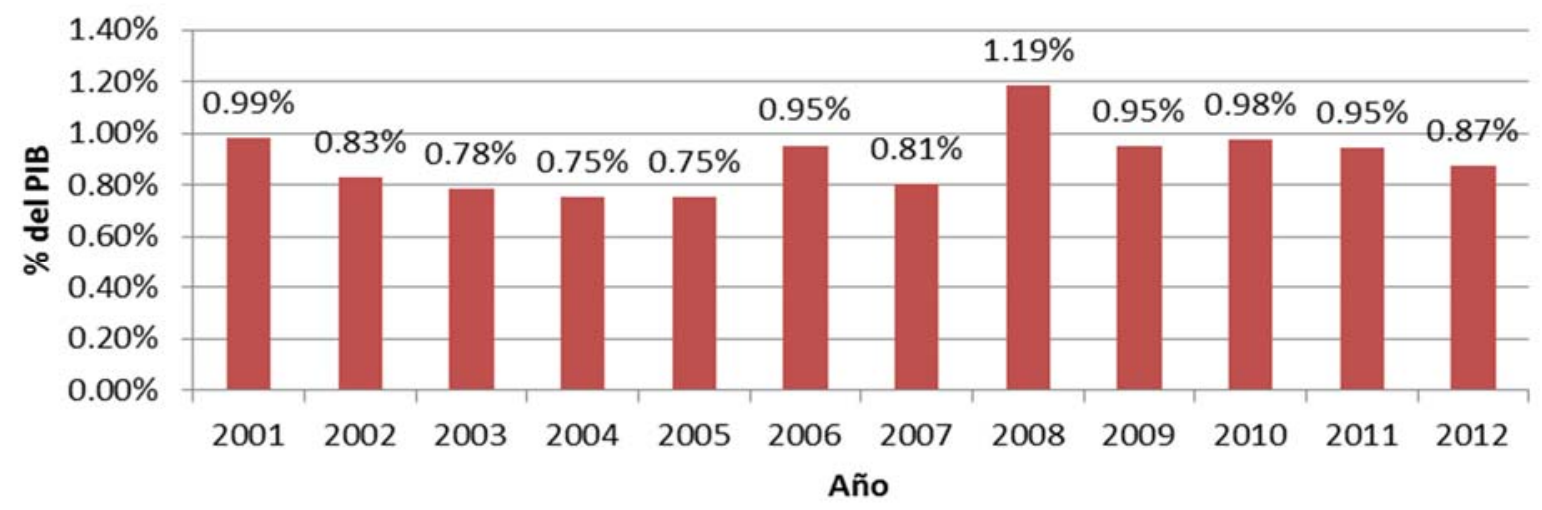

Figura 4. Contribuciones al fondo de pensiones en México (\% del PIB), durante el periodo 2001-2012.

Fuente: Elaboración propia con base en OECD Global Pension Statistics, 2013. La interrupción de la serie en 2006 se debe a la inclusión de los planes de pensiones de empleo registrados por CONSAR desde 2005, no está incluido en los años anteriores. Las contribuciones totales incluyen las contribuciones obligatorias para la jubilación de los empleados, empleadores y el gobierno, y las contribuciones voluntarias y las transferencias del sistema de pensiones anterior (válido hasta el 1997).

En la tabla y figura siguiente, se analiza la composición de la asignación de activos de fondos de pensiones para las categorías de inversión de instrumentos emitidos por el gobierno e instrumentos emitidos por el sector privado.

Tabla 5

Asignación de activos de fondos de pensiones de acuerdo a su inversión en México para los años 2001, 2007 y 2012

\begin{tabular}{llll}
\hline Año & $\begin{array}{l}\text { Bonos emitidos por la } \\
\text { Administración Pública }\end{array}$ & $\begin{array}{l}\text { Bonos emitidos por el sector } \\
\text { privado }\end{array}$ & Total \\
\hline 2001 & $89.82 \%$ & $10.18 \%$ & $100.00 \%$ \\
2007 & $77.89 \%$ & $22.11 \%$ & $100.00 \%$ \\
2012 & $77.98 \%$ & $22.02 \%$ & $100.00 \%$ \\
\hline
\end{tabular}

Fuente: Elaboración propia con base en OECD Global Pension Statistics, 2013. Para 2012, los datos se refieren exclusivamente a planes de pensiones personales. 


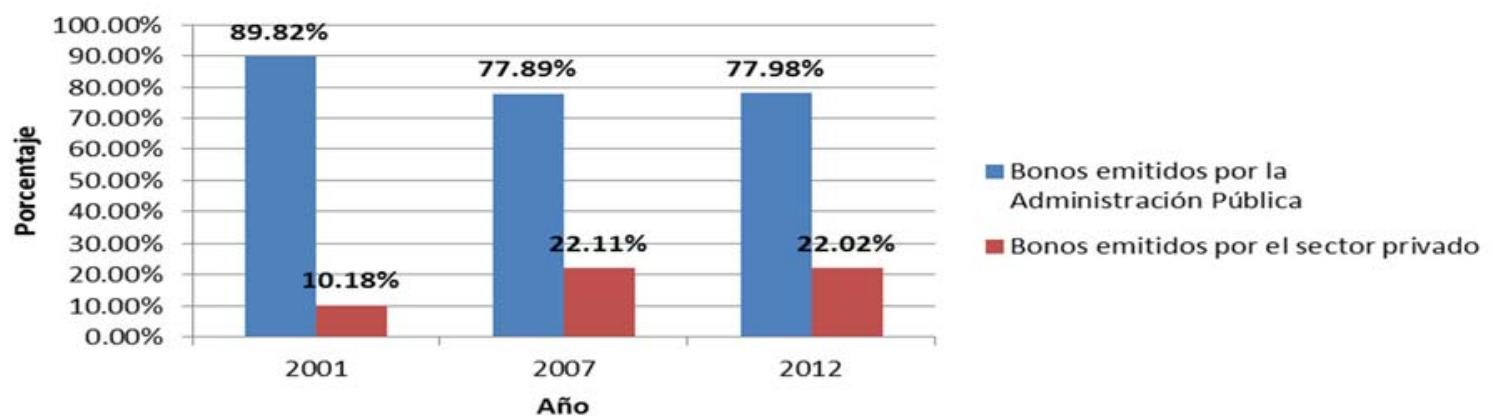

Figura 5. Asignación de activos de fondos de pensiones de acuerdo a su inversión en México para los años 2001, 2007 y 2012.

Fuente: Elaboración propia con base en OECD Global Pension Statistics, 2013. Para 2012, los datos se refieren exclusivamente a planes de pensiones personales.

Los fondos de pensiones se han invertido en bonos públicos y privados, con una composición similar desde 2007, año en que se dio una apertura en cuanto a los instrumentos en que podían ser invertidos estos fondos. Los fondos de pensiones, tanto públicos como privados, han tenido en México un crecimiento relevante, especialmente en las AFORE's. En la inversión de los fondos de pensiones en México, la inversión en bonos tiene un poco más del cincuenta por ciento del portafolio, seguido de bienes y raíces, acciones y, por último, al capital de riesgo. Si en México se quiere desarrollar la industria, la creación de empleos y el crecimiento sostenido, se debe poner énfasis en el tema de la inversión de los fondos de las AFORE'S.

\section{CONCLUSIONES}

El análisis económico nos indica que las personas que requieren de estos servicios crecen a una velocidad mayor que los recursos necesarios para satisfacer estas demandas. Es, en este contexto, donde se encuentra el reto del Estado para poder lograr una cobertura total de estos servicios sin poner en riesgo la sostenibilidad de las finanzas públicas. La implementación de las AFORE's y el paso a las cuentas individuales, fue un buen comienzo por lo cual se sugiere que los fondos de las pensiones sean invertidos en una cartera más amplia de instrumentos financieros, donde se pueda obtener un mayor rendimiento de los mercados financieros y de capitales. En México, donde aún se están resolviendo los temas relacionados a la equidad de género, para el 2005 las mujeres 
tenían una tasa de reemplazo de pensiones menor en 14\% a la de los hombres, para el 2007, la diferencia disminuyó al 6.1\%. Ya sea para hombres o mujeres, para el 2013, la tasa en México era del 44.7\%; es decir, que por cada 100 pesos que ganaba una persona en su etapa productiva, como anciano sólo recibe ingresos de 44.7 pesos. Algo que preocupa los niveles de consumo de los adultos mayores es que los rendimientos promedios de sus carteras de pensión disminuyan anualmente, pasando del $23.59 \%$ en el 2000 a un rendimiento del $11.52 \%$ en el 2014. El Estado debe disminuir la economía laboral sumergida, permitiendo que el paso a la legalidad sea sencillo y estos trabajadores y sus familias tengan estímulos para adherirse al sistema de Seguridad Social; aprovechando además el impacto de la reforma fiscal emprendida por el Ejecutivo Federal en el año 2013. Si no se realizan las acciones necesarias, México puede entrar en una crisis financiera por el funcionamiento actual del sistema de pensiones. 


\section{REFERENCIAS BIBLIOGRAFICAS}

Centro de Estudios de las Finanzas Públicas. (2009). Evolucion de los precios de la canasta basica y su impacto en los salarios y el empleo. Mexico, D.F.: Camara de Diputados del H. Congreso de la Union.

CONSAR. (2013). ¿Que factores determinan mi pension? Una vision sobre las tasas de reemplazo. México: Comisión Nacional del Sistema de Ahorro para el Retiro.

Morales, M. (2007). Nueva ley del ISSSTE y pensiones de retiro. Revista Latinoamericana de Derecho Civil, 263-268.

OCDE. (2013). Panorama de las pensiones 2013: Indicadores de la OCDE y del G20. México. Paris, Francia: OCDE.

Sales, C., Solís, F., \& Villagómez, A. (1997). La reforma al sistema de pensiones: El caso Méxicano. Gaceta de Economía, 11-55.

Solís, F., \& Santín, O. (1999). El sistema privado de pensiones mexicano. Cuadernos económicos de ICE, 325-349.

Villagomez, F., \& Hernández, J. (2010). Impacto de la reforma al sistema de pensiones en México sobre el ahorro. Economía Mexicana Nueva Época, 1-5. 